\title{
DEVELOPMENT OF A DECENTRALIZED LOGISTICS CONTROLLING CONCEPT
}

\author{
Peter Nyhuis, Felix Wriggers, Andreas Fischer \\ Institute of Production Systems and Logistics, University of Hannover, Germany, e-mail: \\ wriggers@ifa.uni-hannover.de
}

\begin{abstract}
Nowadays, companies have to achieve a high logistics performance combined with low costs and high quality. Conventional, resource-oriented production planning and control (PPC) is unable to meet these demands. Both research publications and industrial projects show that logistics controlling based on logistics principles is an effective instrument for improving PPC. A newly developed controlling concept that increases the quality of production planning and control in a significant way is presented in this paper.
\end{abstract}

Key words: logistic controlling; performance measurement

\section{INITIAL SITUATION}

In today's consumer markets, enterprises are increasingly being forced into adapting their products to changing customer demands, promoting technological developments and fulfilling the high requirements placed on delivery lead time and reliability, quality and costs [Wil04].

Therefore, enterprises have to redesign their products and processes in such a way that profitable production will be possible under present and future boundary conditions [Schu97]. Only goal-oriented reproduction, evaluation and interpretation of production processes expose weak spots and help detect means of improvement [Wie02, Sc.Wo99].

Methods of reproduction and analysis that can be generally applied to the order flow are especially scarce for manufacturing processes. The conventional, mere resource-oriented reproduction of manufacturing processes will not be able to cope with the anticipated demands due to lack

Please use the following format when citing this chapter:

Nyhuis, Peter, Wriggers, Felix, Fischer, Andreas, 2006, in International Federation for Information Processing (IFIP), Volume 207, Knowledge Enterprise: Intelligent Strategies In Product Design, Manufacturing, and Management, eds. K. Wang, Kovacs G., Wozny M., Fang M., (Boston: Springer), pp. 399-405. 
of information. Production planning and control can be effectively supported by suitable controlling concepts that are based on reproduction of both, the resource view and the order view. An appropriate controlling concept allows the analysis, evaluation and design of a manufacturing process and helps to manage the lack of information. Thus, the objective of a research project at the Institute of Production Systems and Logistics was to develop a decentralized, participative logistics controlling concept utilizing "intelligent" transponders. Further, employees should be integrated into the logistics controlling. Based on key figures which are calculated decentralized, they shall be qualified to take actions independently to improve processes logistically.

\section{DEFINITION OF THE CONTROLLING OBJECT}

Experience in designing company departments has shown that it makes sense to create, preferably, simple images of the observed departments to begin with. This decreases the complexity of the controlling object and thus increases transparency. Therefore, the controlling object consists of four work systems which are interrelated by so-called "1:1 relationships (as shown in Fig. 1).

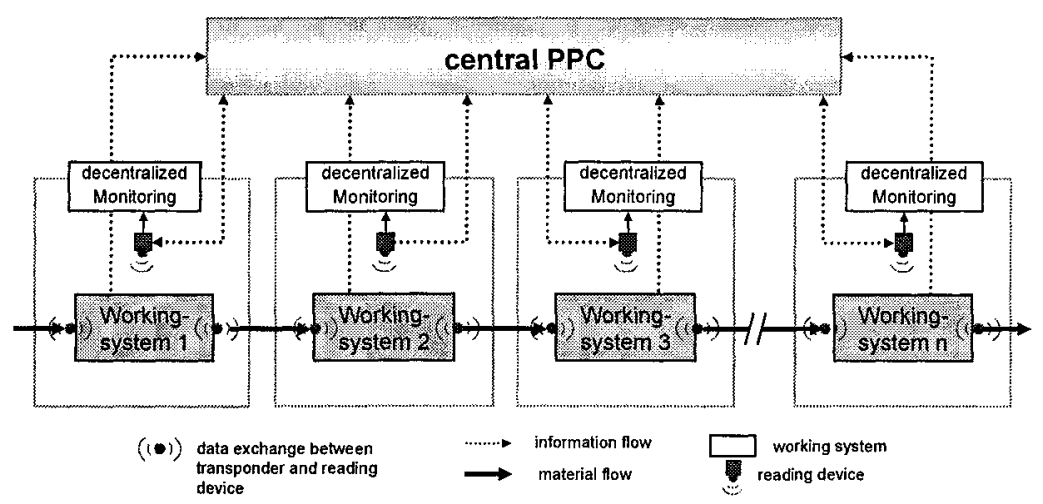

Figure 1 . Controlling object of the decentralized participative controlling concept

The work systems of the controlling object are to be equipped with a computer unit that allows decentralized monitoring. The data required will be captured and passed on by "intelligent" transponders. In this context, "intelligent" transponder refers to a pre-processing label (PPL), providing functions for local calculation of order-specific key data, which has been developed by the Institute for Transport and Automation Technology. 
Key figures derived from business operations form a fundamental basis for controlling. Their task is the focused registration of those facts and trends that can be quantified and thus enable reliable statements. The intention of this specific form of information is to describe complicated facts in a simple way. The significance of key figures is not only determined by the kind of values used for their generation but also by their form of presentation.

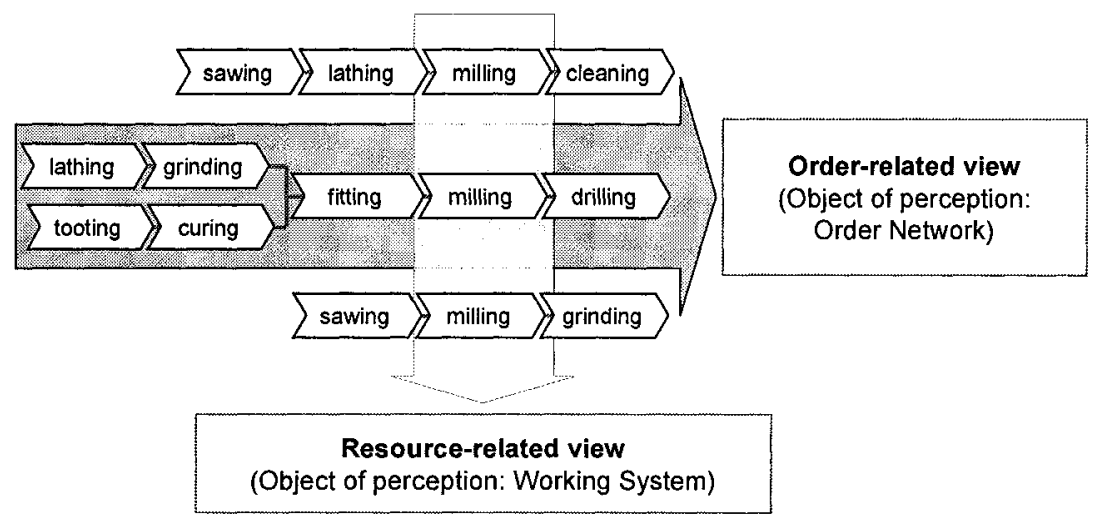

Figure 2. Differentiation between resource-related and order-related view

The performance measurement system of the innovative controlling concept is intended to form the basis for the order- and resource-oriented analysis. The two general perspectives of controlling are related through the individual work processes (see Fig. 2). The individual work process therefore represents a unified basis for all perspectives, which establishes the fundamental requirements for a holistic controlling concept.

\section{RESOURCE-RELATED MONITORING}

Within the monitoring system, the results should be displayed in a clear and straightforward way. Like for the performance measurement system, we distinguish between a resource-related and an order-related representation.

The funnel model describes manufacturing processes by means of the input and output behavior of the participating systems [Wie97]. It facilitates the qualitative and exact-time representation of the dynamic behavior of manufacturing systems and represents the interrelationships between the logistics key figures for throughput time, schedule variance, mean inventory and capacity utilization in the form of characteristic operating curves and makes them accessible for mathematical description [Ny.Wi03].The funnel model and the theory of logistics operating curves have not only proved 
themselves in science, they also have been successfully applied in industry projects to improve logistics performance. Moreover, the model has been incorporated into commercial ERP software and has found applications in controlling. For this reason, the representation of logistical interrelations by means of characteristic operating curves is used in resource-related monitoring. The characteristic operating curves for delivery reliability and throughput time represent the logistics performance (Fig. 3, left), those for performance and costs reflect the logistics expense situation (Fig. 3, right).

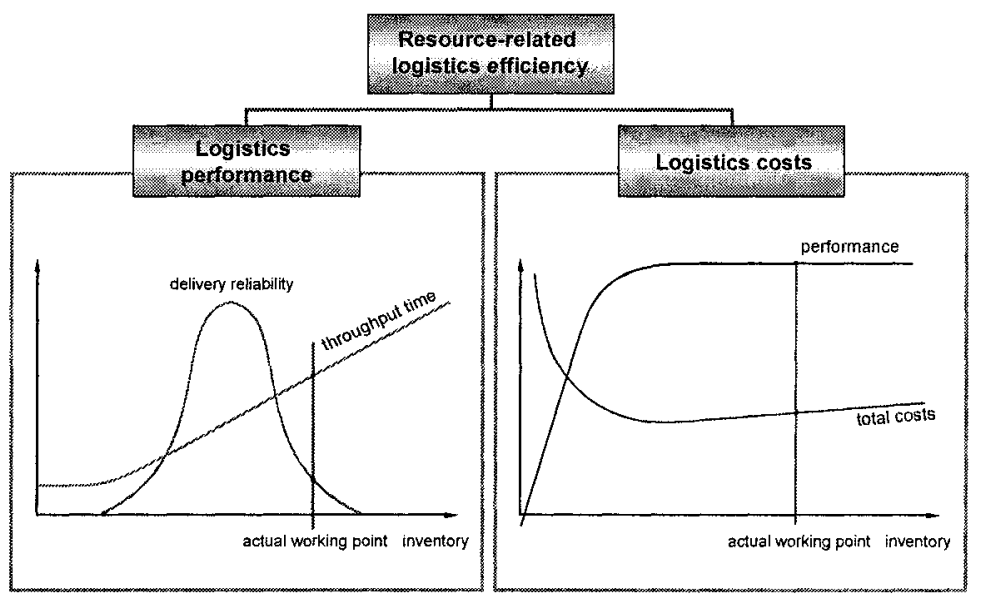

Figure 3. Resource-related logistics monitoring

\section{ORDER-RELATED MONITORING}

The aggregated, order-related key figures for logistics performance and logistics costs are displayed in a different way, because the order reference does not permit a direct connection between these key figures and the inventory level.

The order-related logistics performance can be reproduced by a progress graph (Fig. 4, left). The actual time is plotted as completion confirmation data at defined measuring points and is then compared with the target. Based on this, the scheduling delay can be displayed. This form of representation facilitates the analysis of where and to what extent the delay occurs. This helps users to respond quickly with appropriate countermeasures. The orderrelated logistics costs are illustrated in a cost-time diagram (Fig. 4, right). This diagram displays manufacturing costs and capital tie-up costs, with actual and target values in direct comparison. 


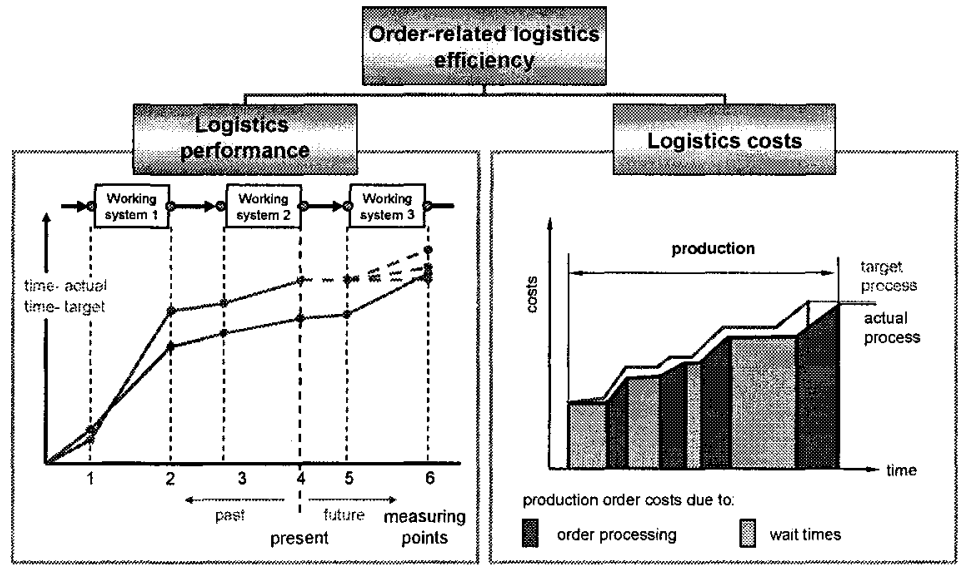

Figure 4. Order-related logistics monitoring

\section{DEFINITION OF MEASURES}

It is necessary to discuss at what time which measures have to be taken and who has to initiate them. The intention is to adjust the load or the capacity in such a way that the target values can be achieved as far as possible. Basic measures for adjusting load and capacity have been subsumed under the concept of capacity adjustment in Fig. 5.

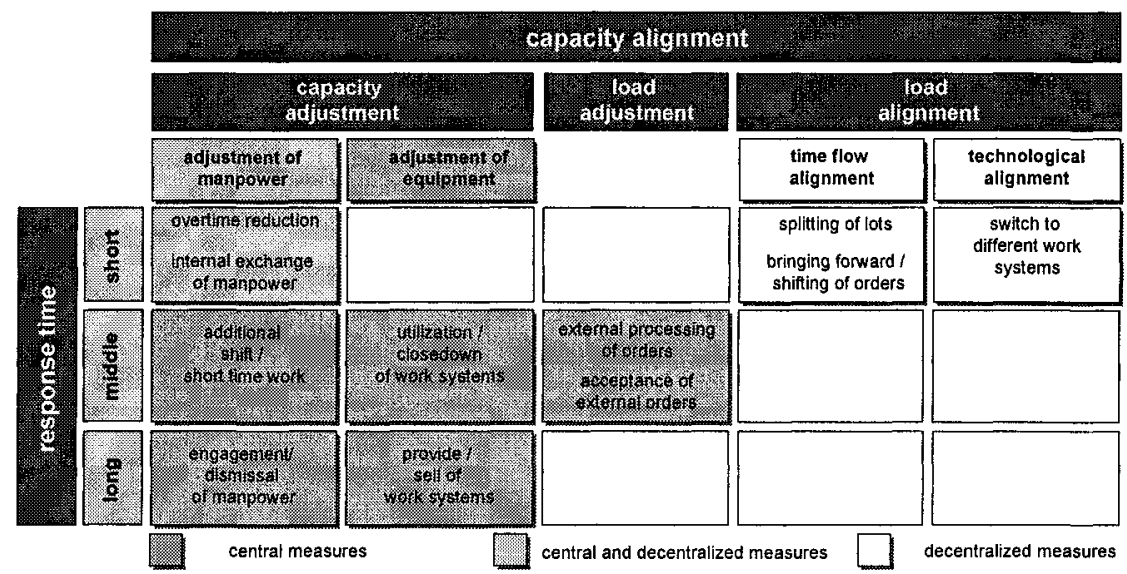

Figure 5. Differentiation of measures for capacity alignment [Büchel] 
The response time needed for the realization of measures is an important criterion for the decision which measures should be taken. A short response time necessitates decisions at plant level. Thus, they can be implemented faster and more flexibly and the employees are integrated into the process. Employees can only participate if measures come under their sphere of competence and influence. This is true for short-term measures only. Medium-term or long-term measures require an interdisciplinary perspective that a production employee usually does not sport. Consequently, decisions regarding long-term and medium-term actions have to be taken centrally.

\section{CONCLUSIONS}

To meet the high demands placed on delivery lead time and reliability, quality and costs, the reproduction, analysis and interpretation of logistics processes are becoming increasingly important. To fulfill these requirements, PPC can be supported by suitable controlling concepts. However, analyses have shown that current controlling concepts possess deficits. For this reason, the Institute of Production Systems and Logistics has developed a new decentralized participative controlling concept based on "intelligent" transponders that decreases these deficits and so increases PPC quality. In the next step the results of this research work will be transferred to assembly orders with integral buffers, finally to whole supply chains.

The authors would like to thank the German Research Foundation for sponsoring this research project.

\section{REFERENCES}

1. [Ny.Wi03] Nyhuis, P. and Wiendahl, H.-P., (2003), Logistische Kennlinien. Grundlagen, Werkzeuge und Anwendung. Springer-Verlag, Berlin,.

2. [Sc.Wo99] Scholz-Reiter, B. and Wolf, H., (1999), Logistik-Controlling: Mobilisierung von Optimierungspotenzialen durch Transparenz in der Logistik. Industrie Management 15.

3. [Schu97] Schuh, G. and Wiendahl, H.-P. (Hrsg.), (1997), Komplexität und Agilität; Springer Verlag, Berlin,.

4. [Wie02] Wiendahl, H.-P., (2002), Erfolgsfaktor Logistikqualität: Vorgehen, Methoden und Werkzeuge zur Verbesserung der Logistikleistung. 2. Aufl. Hans-Peter Wiendahl (Hrsg.), Springer-Verlag, Berlin,. 
5. [Wie97] Wiendahl, H.-P., (1997), Fertigungsregelung. Logistische Beherrschung von Fertigungsabläufen auf Basis des Trichtermodells. Carl Hanser Verlag, München, Wien,.

6. [Wil04] Entwicklungstrends in der Automobil- und Zulieferindustrie. TCW Transfer-Centrum GmbH \& Co. KG, ISBN 3-934155-85-5, München, 2004 